\title{
The optical photometric and spectroscopic investigation of Type IIP supernova 2012A
}

\author{
Rupak Roy ${ }^{1}$, Firoza Sutaria ${ }^{2}$, Subhash Bose ${ }^{1}$, Sean Johnson ${ }^{3}$, \\ Vikram Dwarkadas ${ }^{3}$, Brian York ${ }^{4}$, Brijesh Kumar ${ }^{1}$, Brajesh Kumar ${ }^{1}$, \\ Vijay K. Bhatt ${ }^{1}$, Sayan Chakraborti ${ }^{5}$, Don York ${ }^{3}$, Adam Ritchey ${ }^{6}$, \\ Gabrielle Saurage ${ }^{7}$ and Mary Beth Kaiser ${ }^{8}$ \\ ${ }^{1}$ Aryabhatta Research Institute of Observational Sciences, Nainital, India \\ ${ }^{2}$ Indian Institute of Astrophysics, Bangalore, India \\ ${ }^{3}$ University of Chicago, Chicago, USA \\ ${ }^{4}$ Space Telescope Science Institute, Baltimore, USA \\ ${ }^{5}$ Institute for Theory and Computation, Harvard, USA \\ ${ }^{6}$ University of Washington, Seattle, USA \\ ${ }^{7}$ Apache Point Observatory, Sacramento Mountains, Sunspot, USA \\ ${ }^{8}$ Johns Hopkins University, Baltimore, Maryland, USA
}

\begin{abstract}
Supernova 2012A was discovered on 7.39UT, January, 2012 in the nearby galaxy NGC 3239 at an unfiltered magnitude of 14.6 and classified spectroscopically as a Type IIP event. Here, we present the optical photometric and spectroscopic follow-up of the event during $14 \mathrm{~d}$ to $130 \mathrm{~d}$ post explosion.
\end{abstract}

Keywords. (Stars:) Supernovae: individual (SN 2012A); techniques: photometric, spectroscopic

\section{Introduction}

Given the considerable development in automated sky survey programs, the detection rate of supernovae (SNe) has increased enormously (Lennarz et al. 2012) in the last few years. In a volumetric study it was found that more than $50 \%$ of all SNe are Hydrogen rich (Type II), of which about $70 \%$ show prolonged plateau (IIP) in their light curves (Li et al. 2011). Type II SNe show diversities in their light curves and spectra. Indeed there are distinct classes - normal and subluminous events - which show different properties, along with several peculiar events like SN 1987A, which resulted from explosion of a blue supergiant star. Following the discovery of SN 2008in (Roy et al. 2011a), a different class of SNe has been suggested with characteristics in-between normal and subluminous Type IIP. SN 2009js is a new entry in this category (Gandhi et al. 2013). The nearby event SN 2012A also showed similar behaviour, and is the prime target of our study.

SN 2012A was discovered on 7.39UT, January, 2012 in the nearby ( $\sim 9 \mathrm{Mpc})$ galaxy NGC 3239 at an unfiltered magnitude of 14.6 (Moore et al. 2012). It was classified as a Type IIP event, with a spectrum similar to that of SN 2004et at about 14d post explosion, confirming its identity as a young Type IIP (Stanishev and Pursimo 2012; Roy and Chakraborti 2012).

\section{Observations and data reductions}

The ground based optical photometric observations were carried out at the 104-cm Sampurnanand Telescope (ST) using Johnson $U B V$ and Cousins $R I$ filters, and also from 

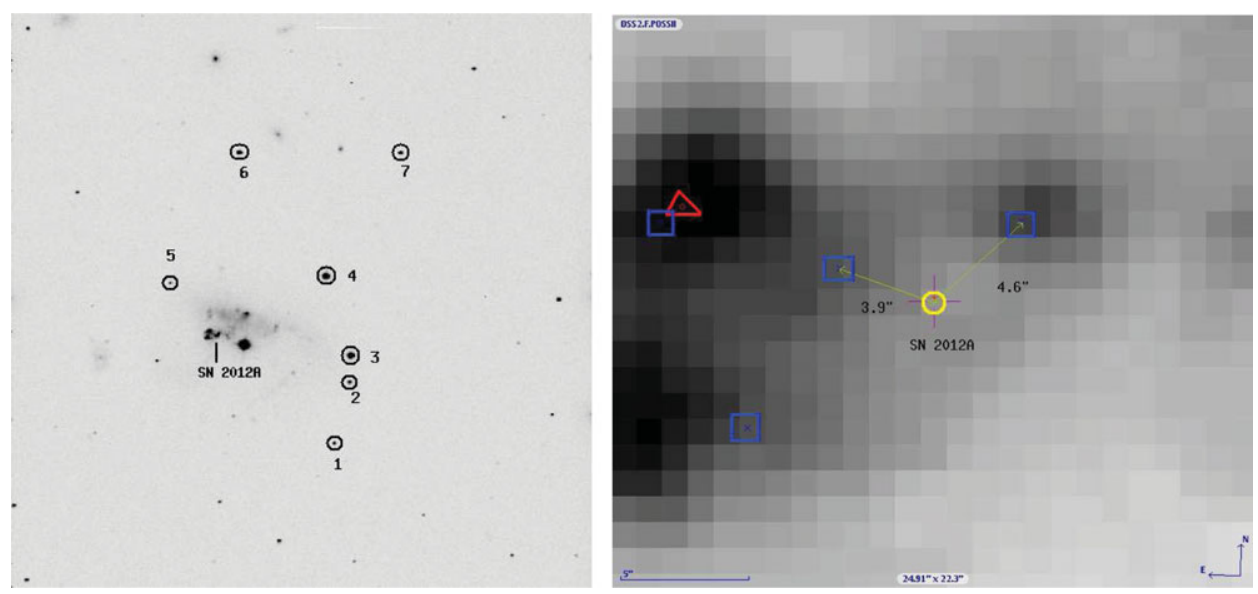

Figure 1. Left Panel: Identification chart of the field of SN 2012A in NGC 3239. The image is about $13^{\prime} \times 13^{\prime}$ taken in $B$-band with the $104-\mathrm{cm}$ ST at ARIES, Nainital. The SN location is marked along with the secondary stars used for calibration. North is up and East is to the left. Right Panel: $24 .{ }^{\prime \prime} 91 \times 22 .{ }^{\prime \prime} 3$ region around SN 2012 A in pre-SN DSS image. The SN location is at the center and marked with the yellow circle. The red triangle is an extended source, whereas the blue rectangles are point sources tabulated in 2MASS catalog. The angular separation between SN and two nearby sources are respectively $3 .^{\prime \prime} 9$ and $4 .{ }^{\prime \prime} 6$. These are the prime sources of contamination of SN flux.

the 130-cm Devasthal Fast Optical Telescope (DFOT) using BVR filters. Photometric observations were conducted at 20 epochs between 14d and 350d. The field of SN 2012A is calibrated using Landolt (1992) standard stars of the fields of SA98. Left panel of Fig. 1 shows the SN position and location of 7 local standards in the field. The detailed methodology for data analysis is discussed elsewhere (Roy et al. 2011a,b). A closer view of the transient location obtained from a pre-SN DSS image is presented in the right panel of Fig. 1. Several star-forming knots, cataloged in the 2MASS survey and marked around the SN position, are prime sources of contamination of SN flux. In order to calculate the correct SN flux, a 'template-subtraction technique' is required, but due to absence of preSN $U B V R I$ images of this field, in the present work the instrumental magnitudes of the SN are derived by the profile (PSF) fitting method. SDSS magnitudes of the nearest star forming knot have been used for a rough estimation of background flux. Therefore the measurements are expected to represent closely the true SN magnitude when it is bright, i.e., in the early plateau phase, while in later epochs the background flux is substantial, and hence the estimated parameters will be marginally affected.

The long-slit low-resolution spectroscopy in the optical range $(0.40-0.95 \mu \mathrm{m})$ was performed at eight epochs during 14d and 134d: three epochs from the 2-m IUCAA Girawali Observatory (IGO), one epoch from the 3.5-m Astrophysical Research Consortium (ARC) telescope and at five epochs from 2-m Himalayan Chandra Telescope (HCT). We also report on the high resolution spectroscopy from 3.5-m ARC at the early plateau phase of the transient. The methodology for spectroscopic data analysis is same as described in Roy et al. $2011 \mathrm{~b}$.

\section{Results}

The calibrated light curves of SN 2012A in $U B V R I$ bands are presented in the left panel of Fig. 2, and its comparison with that of the normal Type IIP SN 2004et (Sahu 

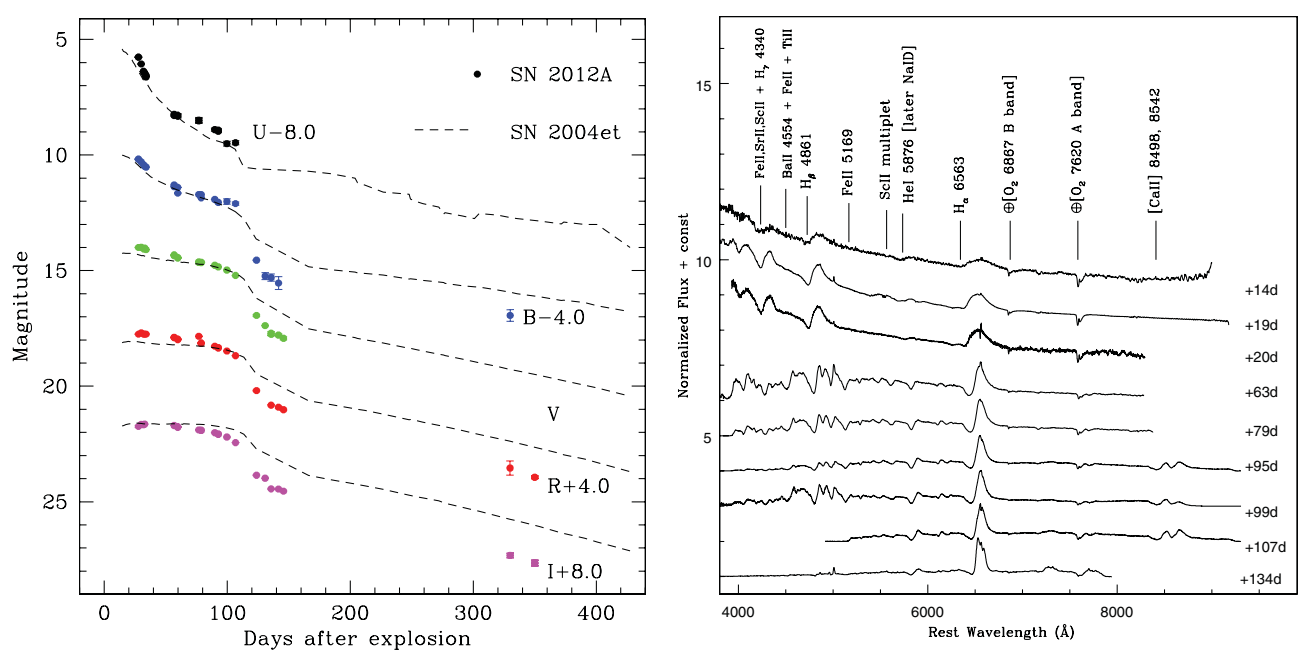

Figure 2. Left Panel: Light curves of SN 2012A in $U B V R I$ bands. The light curves are shifted for clarity, while for SN 2004et they are scaled in magnitude and time to match with SN 2012A. Right Panel: Doppler-corrected (recession velocity $\sim 752 \mathrm{~km} . \mathrm{s}^{-1}$ ) flux spectra of SN 2012A from the plateau (14d) to the nebular phase (134d). Prominent hydrogen and metal lines are marked.
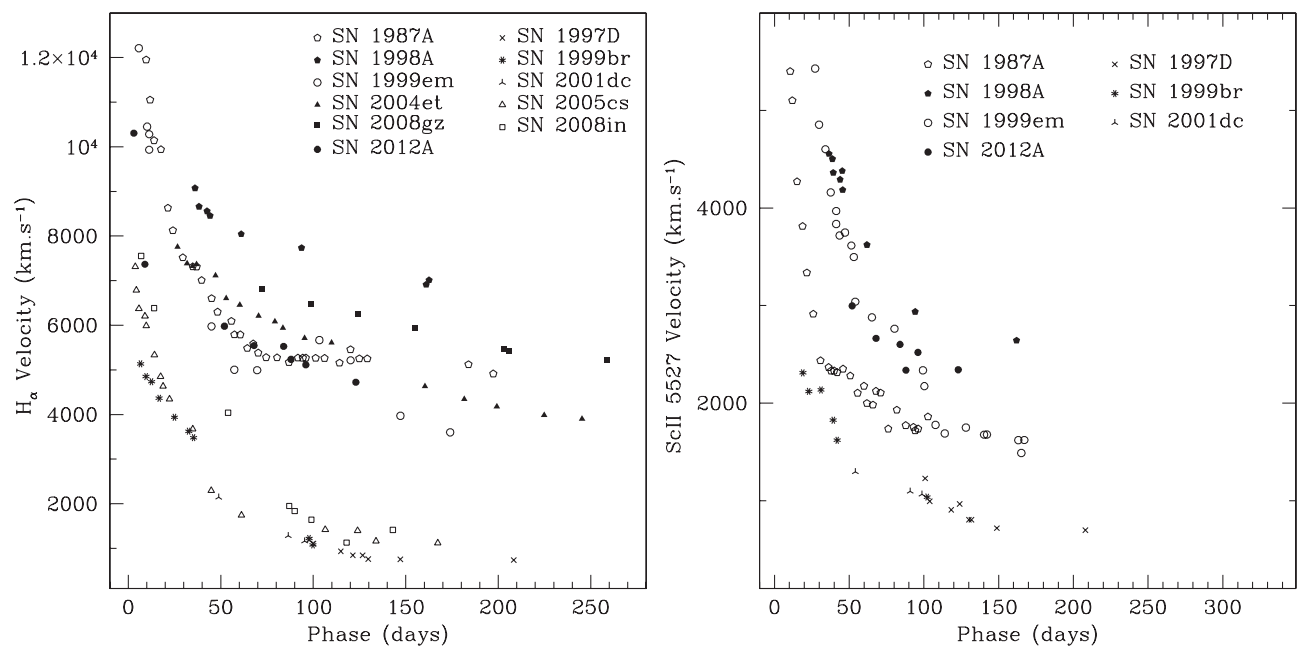

Figure 3. Comparison of velocity profile of SN 2012A with other Type IIP SNe. Left Panel: $\mathrm{H}_{\alpha}$ velocity, showing the velocity near outer ejecta. Right Panel: ScII 5527 velocity, showing the velocity near the photosphere. Ref: Elmhamdi et al. (2003a), Pastorello et al. (2004, 2005, 2009), Roy et al. (2011a, b), Sahu et al. (2006).

et al. 2006) shows that the plateau luminosity of both events decreases in similar fashion, though plateau to nebular conversion is more rapid for SN 2012A than SN 2004et. In nebular phase SN 2012A becomes much fainter than SN 2004et.

The right panel of Fig. 2 shows the evolution of rest-frame spectra of SN 2012A, where the prominent lines of Hydrogen, Helium and metal lines along with several telluric absorption lines have been marked. The line identification has been done using Leonard et al. (2002). The continuum dominated early spectra (14d,19d and $20 \mathrm{~d})$ demonstrate the high photospheric temperature during the initial epochs. The spectral evolution is similar to normal Type IIP SNe. 
Fig. 3 depicts a comparison of outer ejecta and photospheric velocities of SN 2012A with other Type IIP SNe. $\mathrm{H}_{\alpha}$ profiles, representing the velocity of the outer ejecta, show a clear bimodal distribution: low luminosity events asymptotically attain a lower velocity than normal events, though they are much more systematic than normal Type IIP. SN 2012A is located near the lower edge of the velocity distribution of normal events. Photospheric velocity measured from the ScII 5527 line is almost similar in both cases, and SN 2012A preserves the characteristic features of normal Type IIP SNe.

Galactic reddening along the line of sight, $\mathrm{E}(B-V)=0.32 \pm 0.0005$ mag (Schlegel et al. 1998) and the mean distance of the host is $8.6 \pm 0.5 \mathrm{Mpc}$. In our analysis of the high-resolution $3.5 \mathrm{~m}$ ARC spectra, no sign of NaID at host galaxy velocity is found, although there is weak CaII $\mathrm{H}$ and $\mathrm{K}$ absorption. The upper limit on the column density ratio of $\mathrm{NaID} / \mathrm{CaII}$ at the host galaxy velocity is very low ( $\mathrm{NaID} / \mathrm{CaII}<0.04$ ), indicating very little dust grain depletion of Ca. Therefore we presume that extinction is mainly dominated by the Milky-Way.

From the estimated mid-plateau absolute V-band magnitude ( -15.1 mag), photospheric velocity $\left(\sim 2600 \mathrm{~km} \mathrm{~s}^{-1}\right)$ and plateau duration $(\sim 110$ days $)$, the basic parameters of the progenitors have been calculated using the prescriptions of Elmhamdi et al (2003b) and Litvinova and Nadezhin (1985). The amount of synthesized ${ }^{56} \mathrm{Ni}$ is $\sim 0.012$ $\mathrm{M}_{\odot}$, ejected mass is $\sim 26 \mathrm{M}_{\odot}$, explosion energy is $\sim 7 \times 10^{50} \mathrm{erg}$, and the radius of the pre-SN progenitor is about $86 \mathrm{R}_{\odot}$. Assuming the mass of the compact remnant $\sim 2 \mathrm{M}_{\odot}$, main sequence mass of the progenitor can be constrained to be around $29 \mathrm{M}_{\odot}$. This is certainly a crude estimation and more detailed modelling is essential.

\section{Acknowledgements}

We thank George Wallerstein for providing us one of the APO echelle spectra.

\section{References}

Elmhamdi, et al. 2003a, MNRAS, 338, 939

Elmhamdi, et al. 2003b, A\&\&A, 404, 1077

Gandhi, P., et al. 2013, ArXiv, 1303.1565

Landolt, A. U., 1992, $A J, 104,340$

Lennarz, D., Altmann, D., \& Wiebusch, C., 2012, A\&A, 538, A120

Leonard, D. C., et al. 2002, PASP, 114, 35

Litvinova, I. Y. \& Nadezhin, D. K., 1985, Soviet Astronomy Letters, 11, 145

Li, W., et al. 2011, MNRAS, 412, 1441

Moore, B., Newton, J., \& Puckett, T., 2012, CBET, 2974, 1

Pastorello, A., et al. 2004, MNRAS, 347, 74

Pastorello, A., et al. 2005, MNRAS, 360, 950

Pastorello, A., et al. 2009, MNRAS, 394, 2266

Roy, R., et al. 2011a, ApJ, 736, 76

Roy, R., et al. 2011b, MNRAS, 414, 167

Roy, R. \& Chakraborti, S., 2012, CBET, 2975, 2

Sahu, D. K., Anupama, G. C., Srividya, S., \& Muneer, S., 2006, MNRAS, 372, 1315

Schlegel, D. J., Finkbeiner, D. P., \& Davis, M., 1998, ApJ, 500, 525

Stanishev, V. \& Pursimo, T. 2012, CBET, 2974, 3

\section{Discussion}

Bersten: The physical parameters found for supernova 2012A seems somewhat inconsistent. With a ejecta mass of $24 M_{\odot}$ and the very small energy found, the plateau should be extremely long. 
Roy R.: This work is preliminary. We have applied LN85 to calculate the burst parameters. Detailed calculations with more improved theoretical models are in progress.

Nomoto: A comment: The relation between the $\mathrm{H}$ velocity and the luminosity you showed may be interpreted as follows: The $\mathrm{H}$-alpha velocity is a good indicator of mixing, thus suggesting the progenitor mass as follows. The lower $\mathrm{H}$ velocity means a larger scale of mixing of $\mathrm{H}$ down to the deeper layer. Such a deep mixing occurs in smaller mass progenitor because more massive envelope (due to less mass loss) causes a stronger Rayleigh-Taylor instability. The smaller mass progenitor tends to be fainter. The higher $\mathrm{H}$ velocity suggests more massive one. 